\title{
Tangence
}

\section{Paulin Gagne, le poète qui faisait rire de lui}

\section{Pierre Popovic}

Numéro 53, décembre 1996

L’humour de la poésie

URI : https://id.erudit.org/iderudit/025928ar

DOI : https://doi.org/10.7202/025928ar

Aller au sommaire du numéro

Éditeur(s)

Tangence

ISSN

0226-9554 (imprimé)

1710-0305 (numérique)

Découvrir la revue

Citer cet article

Popovic, P. (1996). Paulin Gagne, le poète qui faisait rire de lui. Tangence, (53), 76-101. https://doi.org/10.7202/025928ar d'utilisation que vous pouvez consulter en ligne.

https://apropos.erudit.org/fr/usagers/politique-dutilisation/ 


\section{Paulin Gagne, le poète qui faisait rire de lui Pierre Popovic}

Il existe des fous simples et puis il existe d'autres fous, ceux que torture la marotte de la civilisation...

Louis-Ferdinand Céline, Voyage au bout de la nuit

"Salvat ridendo mundum", il sauve le monde en riant, est ma devise.

Paulin Gagne, L'Archi-Monarquéide ou Gagne Premier

Paris, au mitan du $\mathrm{XIX}^{\mathrm{e}}$ siècle, est une ville bruyante et spectaculaire. Qui veut être connu ou reconnu, qui veut être cité dans la "prière du matin de l'homme moderne" (Hegel) qu'est le journal, doit s'y faire entendre et voir. Quand on ne possède ni l'aisance "naturelle" que permet le train de vie bourgeois ni les galons de parade qui assurent son prestige et sa visibilité à l'uniforme militaire, quand le capital social, culturel, financier manque pour épater les galeries de l'Opéra, pour attirer l'attention sur les boulevards ou pour graviter autour des arènes du pouvoir et du savoir, quand "la vie parisienne " ${ }^{1}$ n'est pas "spontanément" disposée à accueillir vos aspirations légitimes, il faut trouver des solutions autres, des tactiques surprenantes, des moyens inouïs pour ne pas disparaître, esprit et corps, dans la mêlée. D'aucuns cherchèrent ces moyens dans le travail, dans le crime, dans l'épargne, dans cette "lutte pour la vie "à laquelle s'adonnent tant de héros romanesques, du Rastignac conquérant du Père Goriot au Mouret parvenu du Bonbeur des dames: d'autres, mûs par quelque ressort romantique, choisirent l'art ou la littérature. Il en fut aussi qui trouvèrent ces moyens dans le défrayage systématique de chronique, adoptant des comportements socialement

1 Cf. Daniel Oster et Jean Goulemot, La vie parisienne. Anthologie des maeurs du $x x^{e}$ siècle, Paris, Sand/Conti, 1989, $354 \mathrm{p}$. 
hétérodoxes, déplacés, inordinaires. Aussitôt jugés iconoclastes ou dérangés, vite tenus pour de doux rêveurs ou de distrayants illuminés sans lesquels la vie de la capitale n'aurait somme toute pas été ce qu'elle était, ils firent les délices de la rumeur urbaine. C'est à la régalade que les doxographes les élurent rois de la rigolade ou de la saugrenuité. Ils ne sont pourtant pas à part dans l'imaginaire social de la modernité naissante. Ils en sont l'un des rouages. Les "originaux", les "excentriques", les "singuliers" constituent une pièce d'angle de la représentation de la ville et un lieu commun très couru du discours public. Leur exemple est utilisé pour souligner, par rétroaction, que Paris est bien la ville moderne par excellence: ils sont la preuve qu'on y disjoncte avec entrain et que l'originalité n'y possède à ce point pas de frein que sa recherche achève de confondre les esprits par avance faibles ou égarés. Il sert également à justifier par la bande la nécessité de l'ordre. Bon nombre d'articles consacrés aux personnages incongrus de la scène publique présupposent ou laissent entendre cette crainte: que deviendrait la société, la vie, le monde s'ils étaient laissés aux mains de ces irresponsables? Ce texte, paru dans Le charivari à l'occasion des élections de 1876 auxquelles Paulin Gagne présentait sa candidature, en est un bon exemple.:

M. Gagne vient de poser sa candidature dans un arrondissement de Paris.

Il n'est pas constitutionnel, $M$. Gagne, car il se proclame candidat archi-monarchiste-impérialiste.

Je trouve que jusqu'à ce jour les candidatures archi-amusantes sont rares.

Pourquoi n'entend-on plus parler de M. Bertron, le candidat humain?

M. Bertron me manque. Je demande M. Bertron.

Je ne propose pas une récompense honnête à qui me le rapportera; c'est un simple appel bienveillant que je fais.

Il ne faut pas plaisanter avec les candidats comiques.

Un jour M. Gagne pourrait bien finir par être élu.

Supposons, en effet, que dans un conflit électoral les habitants d'une circonscription fussent très embarrassés dans le choix d'un candidat et que chacun, pour s'amuser, jetât dans l'urne un bulletin au nom de $M$. Gagne.

Ce candidat universel pourrait ainsi, au premier tour de scrutin, obtenir la majorité des suffrages...

Et M. Gagne irait siéger à l'Assemblée. 
Cette plaisanterie serait drôle si elle ne devait pas durer quatre ans. ${ }^{2}$

À l'instar du remarquable Adolphe Bertron, Paulin Gagne fut l'un de ces individus dont les hauts faits aujourd'hui oubliés alimentèrent le discours public parisien entre 1841 et 1876 . "Candidat universel "à Paris aux élections de 1863, 1868 et 1876, "avocat des fous" et "ex-bâtonnier de l'ordre ", fondateur, directeur et seul rédacteur de feuilles éphémères destinées selon lui à apporter un peu de claire clarté sur la noire obscurité de ce monde (Le théâtre du monde, Le joumalophage), confident régulier et courroie de transmission occasionnelle de l'Esprit divin, miraculé par autoproclamation, exécuteur d'intenses séances de prosternation aux alentours de La Madeleine et de Notre-Dame des Victoires, inventeur et inlassable propagandiste de la généreuse "philanthropophagie" ${ }^{3}$, dénonciateur éminemment moral des effets néfastes du suicide et du danger des voyages en chemin de fer, "vainqueur de la bataille de l'Obélisque " ${ }^{4}$, Gagne fournit à Gill, Cham, Job et

2 Adrien Huart, "Chronique du jour", Le charivari, 9 février 1876, [p. 34].

3 Il s'agit de la plus haute idée humanitaire de Paulin Gagne: "La Philanthropophagie, faite par le sacrifice volontaire des hommes et des femmes, [consiste à se livrer] fraternellement et religieusement en nourriture aux victimes de la faim qui dévore le monde..." (cité par André Blavier, Les fous littéraires, Paris, Henry Veyrier, 1992, p. 697). Gagne vit dans la période de pénurie que fut le siège de Paris une occasion idéale pour démontrer la justesse de ses convictions et proposa que soient philanthropophagés tous les vieillards de plus de soixante ans. Nul ne pouvait l'accuser de manque d'altruisme, puisqu'il venait lui-mème d'atteindre soixante-deux ans, mais il ne fut pas suivi. De façon générale, le cannibalisme et le fantasme de la dévoration constituent deux leitmotive de l'œuvre gagnéenne.

4 L'affaire est racontée en ces termes par Victor Fournel: "M. Raspail ayant déclaré qu'il se rendrait au Corps législatif, dut-il être seul, pour réclamer l'ouverture immédiate de la session, $M$. Gagne fit savoir par tous les journaux qu'il suivrait le député radical, dut-il être seul aussi, - et qu'il se tiendrait au pied de l'Obélisque, armé d'un petit levier pour ouvrir les portes de la Chambre; - de plus, il donnait rendez-vous à tout le monde, recommandant à ceux qui se présenteraient de ne pas oublier leur petit levier. Arrivé au pied de l'Obélisque, où les personnes qui attendaient le reçurent par des acclamations unanimes, il fit un discours, expliqua le jeu des petits leviers, - il en tenait un caché dans son parapluie, - et termina en disant qu'il allait attendre $M$. Raspail. Le député radical ne parut pas; mais une pluie violente s'étant mise à tomber, la foule se dispersa et $M$. Gagne resta. bientôt seul, comme il l'avait dit, au pied de l'obélisque, abrité sous son parapluie. Ainsi finit une manifestation qui avait semblé un moment pouvoir devenir dangereuse, et qui était surveillée, de loin, par des sergents de ville gardant toutes les issues de la place." (Les rues du Vieux Paris. Galerie 
Bertall des kyrielles de caricatures hilarantes et fut pour la presse et les revues de son temps un inépuisable sujet de réjouissances.

Mais Gagne n'est pas que ce personnage coloré, officiant aux frontières du fait divers cocasse et de la vie politique, devenu défrayeur de chronique professionnel sous le second Empire. Il fut et se voulut avant tout écrivain, mieux: poète. Si les palmarès habituels de l'histoire littéraire ignorent généralement jusqu'à son nom, celui-ci n'est pourtant pas tout à fait inconnu des spécialistes de la poésie française du $\mathrm{XIX}^{\mathrm{e}}$ siècle. Ces derniers le rencontrent quelquefois au hasard des œuvres intimes ou des correspondances des "grands auteurs" (par exemple dans les Euvres postbumes de Baudelaire ${ }^{5}$ ou dans le Journal de Michelet ${ }^{6}$ ) et ils l'aperçoivent toujours, étonnamment voisin de Musset, dans ces vers tirés du poème d'ouverture des Amours jaunes de Tristan Corbière:

... Ça, c'est naivement une impudente pose;

C'est ou ce n'est pas ça : rien ou quelque chose...

- Un chef d'œuvre? - il se peut: je n'en ai jamais fait.

- Mais, est-ce du huron, du Gagne, ou du Musset?

- C'est du... mais j'ai mis là mon humble nom d'auteur

Et mon enfant n'a pas même un titre menteur.

En contrebas de ces vers, quelle que soit l'édition des Amours jaunes consultee, survient toujours une notice semblable:

Paulin Gagne (1808-1876) était un mauvais poète très prolixe qui, entre 1841 et 1875 , amusait le public en rédigeant des journaux en vers dans lesquels il formulait des projets extravagants: la "philanthropophagie *, la " républiquéide-archimonarchie ", etc. $^{\text {p }}$

populaire et pittoresque. Ouvrage illustré de 165 gravures sur bois, Paris, FirminDidot, 1879, 663 p., p. 652). Vu du point de vue de Gagne, il s'agissait néanmoins d'une grande victoire, ce qui revient à dire que la "victoire" dépendait plus pour lui de l'écho médiatique recueili par son fait d'armes, que de la réalisation de celui-ci, laquelle était en l'occurrence plutôt problématique.

5 Cf. Charles Baudelaire, Euvres postbumes, Paris, Mercure de France, 1908, p. 278.

6 Qui reçut sa visite et la note en ces termes: •Visite du mystique Gagne, mari de $M^{\text {me }}$ Élise Moreau, auteur d'Omégar, le dernier homme, de L'Unitéide." (Jules Michelet, Journal. Tome II (1849-1860). Texte intégral, établi sur les manuscrits autographes et publié pour la première fois, avec une introduction, des notes et de nombreux documents inédits par Paul Viallaneix, Paris, Gallimard, 1962, 837 p., p. 496).

7 Notice de Michel Dansel dans Tristan Corbière, Les amours jaunes. Cboix de poèmes, Paris, Librairie Larousse, 1975, p. 21. 
80

Paulin Gagne (1808-1876): mauvais versificateur prolixe et amuseur public qui se présentait comme candidat à toutes les élections ${ }^{8}$.

Cependant, le choix des trois emblèmes dans les vers précités témoigne d'une autodérision typique chez l'auteur des Amours jaunes, autodérision qu'il est certes toujours loisible de tenir pour une bravade pathétique exhibant la désinvolture avec laquelle le jeune poète "crochard" et revêche observe la scène littéraire et la Poésie avec un grand $P$, mais qu'il est moins naif de tenir pour l'inversion sublimée d'une situation précaire dans le champ littéraire: à Paris, Corbière n'a ni les relations éditoriales ni les entrées sociales ni les amitiés littéraires qui lui permettraient un accès facile à la scène restreinte 9 . Les aurait-il eues que le vers précité aurait pu prendre une autre tournure, par exemple celle-ci:

- Est-ce du Hugo, du Baudelaire, du Coppée?

- C'est du... mais j'ai mis là mon humble nom d'auteur

Et mon enfant n'a pas même un titre menteur.

De cette façon, Corbière aurait fermement pris position dans l'espace littéraire environnant, estampillant d'un seul coup les trois adversaires symboliques qui occupent le devant de la scène: la tradition romantique et son indestructible dernier grand mage (Hugo), la poésie moderne en voie de consécration (Baudelaire), l'avant-garde reconnue (Coppée, les Parnassiens). La pose de dégoût qu'il affiche à l'endroit de sa propre poésie - pose qui est un leurre, une forme d'énonciation transformant l'ensemble des Amours jaunes en paradoxe pragmatique - le conduit à prendre position par le bas, à valoriser son excentrement, à faire cohabiter "du huron", "du Gagne", "du Musset". En conjoncture, le "huron" connote le sauvage, le non-civilisé, le brut, et renvoie au fait que Corbière ne respecte ni la langue littéraire (il utilise des mots bretons et de l'argot, pratique l'ellipse sur une grande échelle, adore les calembours soufrés) ni les conventions de la poésie reconnue (il malmène l'alexandrin, ponctue à la hussarde, affectionne les ruptures soudaines de ton et les énoncés allotopes, soumet la prosodie à son rocailleux crin-crin). "Musset" demeure un emblème

8 Autre notice de Michel Dansel dans Rimbaud, Lautréamont, Corbière, Cros. CEuvres poétiques complètes, Paris, Robert Laffont, 1980, 952 p., p. 855.

9 Contrairement à Rimbaud, son exact contemporain (Les amours jaunes et Une saison en enfer datent de 1873), Corbière ne cherche pas à entrer en contact avec les milieux d'avant-garde de la capitale. 
du romantisme, mais d'un romantisme désuet, impuissant, ruiné, avachi, que vient d'achever (dans tous les sens du terme) Baudelaire. À l'exemple des beautés perdues Sur trois marches de marbre rose, Les amours jaunes sont déplacés, anachroniques. Gagne, quant à lui, symbolise l'incongruité, le délire, l'énormité, cette limite où l'original le dispute à l'absurde, le comble du poète exubérant mais raté, le bouffon des lettres.

Cette réputation, il serait difficile de prétendre que Paulin Gagne ne l'avait pas cherchée, et que Corbière le cite est en soi révélateur du capital de pittoresque qu'il avait dans les journaux, les revues et les conversations de brasserie ou de boulevard. Ne commettant jamais moins que quelques milliers de vers sur le moindre sujet de circonstance, il multiplia les cuvres extravagantes avec une constance sidérante ${ }^{10}$ et mérita l'une des plus hautes places chez les collectionneurs de la "folie littéraire", de Philomneste Junior, Raymond Queneau et André Blavier à Guy Bechtel et Jean-Claude Carrière ${ }^{11}$. Dans un résumé commenté de la mâ̂tresse-cuuvre de Paulin Gagne, à savoir les 25000 vers de L'Unitéide, ou la Femme-Messie, poème universel en 12 chants et 60 actes, avec cbœurs, précédé d'un prologue et suivi d'un épilogue par Mme Gagne (Ëlise Moreau de Rus) ${ }^{12}$, Octave Delepierre donne une assez juste idée de la manière gagnéenne. Ce qu'il dit des trente-huitième et trente-neuvième actes du huitième chant suffira à prouver l'étonnement du critique:

L'acte $38 \mathrm{me}$ du chant VIIIme, se développe dans un vaste champ de pommes de terre, et la Pataticulture ouvre la scène par un discours de 72 vers, d'autant plus singuliers que, comme nous le démontrerons tout à l'heure, ils sont écrits très sérieusement : -

Peuples et Rois, je suis la Pataticulture,

Fille de la nature et du siècle en friture;

Jai toujours adoré ce fruit délicieux

10 On trouvera en annexe une bibliographie des écrits de Paulin Gagne.

11 Cf. Raymond Queneau, Les enfants du limon, Paris, Gallimard, 1987 (1938), 316 p. (en ce qui concerne Paulin Gagne, voir p. 273-276); André Blavier, Les fous littéraires, Paris, Henry Veyrier, 1992, 924 p. (en ce qui concerne Paulin Gagne, voir p. 687-713); Philomneste Junior (pseudonyme de Pierre Gustave Brunet], Les fous littéraires, Bruxelles, 1880, in-8; Guy Bechtel et Jean-Claude Carrière, Dictionnaire de la bêtise et des erreurs de jugement. Le livre des bizarres, Paris, Robert Laffont, 1992, 790 p.

12 [Paris], Chez tous les libraires de France et de l'étranger, [1857], in-8, 726 p. 
Que, dit-on, pour extra, mangeaient jadis les Dieux.

La tirade se termine par ce vers:-

Dans la pomme de terre est le salut de tous!

On croirait qu'il est difficile d'aller plus loin dans le grotesque; mais à l'acte suivant, dont la scène, dit le texte, se passe partout, La carotticulture tient aux rois et aux peuples un discours qui l'emporte sur le précédent. On y trouve la parodie de la Marseillaise, intitulée la Carotte universelle, commençant par: -

Allons, Enfans de la Carotte,

Le jour de gloire est arrivé.

Et le choeur chante: -

Aux armes, Carottiers, formez vos bataillons,

Marchons, que la carotte inonde nos sillons.

Probablement que le lecteur croira que tout ceci n'est qu'une plaisanterie; mais non seulement $M$. Gagne est très sérieux, en expliquant son ceuvre, mais il cléclare en outre, dans la préface, que le vaste sujet de ce poème bumanitaire et Chrétien, doit former la poétique universelle de l'bumanité, et l'école de la vérité, et il s'écrie, plein d'enthousiasme: -

Telle est, telle est la Sainte et nouvelle épopée

Que de mon pur amour l'âme a développée!

Ensuite Mme Élise Gagne, sa femme, ajoute un épilogue, où elle proclame qu'après les réformes indiquées dans le poème: -

L'abondance parvint à chasser la misère,

Et le bonheur des cieux habita sur la terre.

L'ensemble prouve, en un mot, que $M$. Gagne a employé toutes les ressources de son intelligence pour écrire ce chef-d'œuvre, et si le lecteur est tenté de rire, c'est qu'il ne comprend pas l'extrême profondeur de la pensée qui enfanta ce poème.

N'est-ce pas bien le cas de dire, avec le sieur de Longval "Lorsque cette Méduse (la manie) s'est une fois glissée dans le cerveau, elle sait si bien offusquer l'imagination, pervertir les pensées, transporter l'esprit, et corrompre la raison, que par son moyen les actions et les paroles des hommes se tournent en extravagances ${ }^{13}$

Il serait pourtant faux de croire que le style et la lettre de l'ouuvre de Gagne ont toujours été tels. Sa première ceuvre, Le Suicide, ou Cris de désespoir, de baine, de défaites, et cbants d'espérance, d'amour, de triomphe, parue en $1841^{14}$, fut celle dans

13 Octave Delepierre, Histoire littéraire des fous, London, Trübner \& Co., 60 , Paternoster Row., 1860, 181 p., p. 61-65.

14 Pour l'essentiel, l'œuvre de Gagne paraît à compte d'auteur, ainsi qu'une immense quantitê d'œuvres du xix ${ }^{\complement}$ siècle, déclassées et tenues pour illégitimes. 
laquelle il plaça tous ses espoirs. En soi, le projet ne manquait pas d'à-propos. Pour une entrée en scène, le thème était plutôt bien choisi : la vague de suicides qui a accompagné le romantisme durant les années trente a frappé les imaginations. Plus largement, de Goethe et Germaine de Staël aux réflexions $A$ propos du suicide de Marx, la mort volontaire est un véritable fascinus de la modernité littéraire et l'on sait combien Chatterton ou les suicides des deux jeunes dramaturges Escousse et Lebras ont entrainé de débats entre les plus grands des romantiques (Hugo, Vigny, Gautier, Musset). Faisant un "poème dramatique ", Gagne joue également la carte du mélange des genres dont Hugo avait fait l'un des axes de l'esthétique nouvelle: Gagne tente d'unir le poème, le drame et le thème d'actualité. Il prend aussi parti pour les poètes, tente de définir leur fonction sociale, stigmatise une société qui ne laissera bientôt plus, selon lui, que deux solutions au poète: l'hôpital ou la mort. Tout cela, bon an mal an, peut prendre sens ${ }^{15}$. On devine même qu'il y a là tout le potentiel pour faire un coup d'éclat ou, au moins, pour trouver une place convenable sur la scène des lettres. Et pourtant, ce fut un échec royal, résolu, définitif.

Michel Pierssens observe que la frontière entre littérature reçue et littérature déclassée est d'abord éditoriale: "Solidement appuyée sur les figures émergentes de l'Éditeur et de l'Auteur, ainsi que sur des catégories devenues prégnantes telles que celles de format ou de collection, nous savons désormais comment la littérature s'est faite institution. Mais ce faisant, bien des formes plus anciennes ou moins balisées du passage à l'imprimé, désormais marginalisées, ont eu tendance à échapper à l'attention historienne ou critique. Toute la floraison d'écrits sauvages qui faisait pour une part le pittoresque imprévisible et l'identité attachante du $\mathrm{XIX}^{\mathrm{e}}$ siècle, mais qui serait aujourd'hui trop excentrique pour garder la moindre chance de voir le jour, pouvait atteindre le stade de l'impression. La toute-puissance matérielle et symbolique de la Maison d'édition moderne a fait oublier l'ancien libraire-imprimeur, tout comme l'auteur isolé qui pouvait choisir de s'y faire éditer à son compte, totalement ou partiellement. Toute une partie de la production de livres est ainsi tombée hors du champ de l'histoire, de même que les cuvres livrées à ce circuit, tombées elles aussi hors du champ de l'esthétique ou de la pensée légitime. La ligne de partage entre la littérature et le reste, entre la science et la pseudo-science, entre philosophie et délire, épouse très largement celle qui distingue l'Édition du Compte d'auteur. " "Fictions célestes", Revue des sciences bumaines, $\mathrm{n}^{\circ} 235$ (octobre-décembre 1994), p. 87-109, p. 87-88).

15 Et n'est pas sans rejoindre un courant de revendications qui, partant de textes comme Les grands poètes morts de faim de Bin de Saint-Victor et la Biographie des auteurs morts de faim de Colnet, aboutit via Stello et Chatterton à la Lettre aux dêputés sur la propriété littéraire que Vigny signe la même année (1841). 
84

Était-ce manque de talent? Oui, assurément, les vers de Gagne ont souvent de ces maladresses ou de ces "fautes de goût" désolantes qui ne peuvent qu'attirer le haro ou le mépris des critiques. Mais, outre qu'il ressort de façon évidente que Gagne est profondément seul, qu'il n'a ni les appuis ni les conseils littéraires qui lui permettraient de trouver le ton, la voix, le propos juste, il y a que son texte tout entier repose sur une stratégie d'échec obvie. En effet, alors qu'il fait preuve d'une réceptivité manifeste aux thèmes, à la sensibilité, à la lyrique romantiques, Gagne inverse la vapeur, devient moralisateur à tout crin, verse dans un antiromantisme qui trouve sa source directe dans cet épisode qu'il relate avec amertume et qui n'a guère sa place dans un "poème dramatique":

Auteur, sans nul espoir, d'une pièce tragique,

Que je trouve, il est vrai, moi-même assez comique,

Je voulus consulter un bienveillant auteur,

Pour en faire estimer à peu près la valeur;

Je me rends $[. .$.$] , un jour, chez mon juge sévère$

Qui me reçoit d'abord comme un digne confrère;

Soudain nous prenons place, et relevant le ton,

Du héros du sujet je décline le nom,

À l'instant, mon auteur, enragé romantique,

Présume que je vais lui lire du classique,

Et se levant alors d'un air tout dédaigneux,

M'annonce que le temps l'appelle en divers lieux,

Me priant de laisser en ses mains mon ouvrage

Qu'il lira seul, dit-il, avec plus d'avantage;

Sans répliquer un mot à ce touchant début,

J'empoche mon écrit et lui fais mon salut ;

Trois jours étaient passés, quand un démon sans doute,

Me le fait nez à nez rencontrer sur ma route,

Il maborde aussitôt, et par un tour maudit,

J'ai lu, mannonce-t-il, "hier ", votre manuscrit,

La scène n'est pas mal, la "prose * est bien écrite,

Continuez, Monsieur, vous avez du mérite;

Mais n'allez pas chercher des Grecs ou des Romains,

Faites-nous du moderne et l'on battra des mains!

À de pareils aveux faits d'un air plein d'audace,

J'étais tout stupéfait, le regardant en face,

Ne sachant si je dois rire au nez du bourreau,

Ou d'un grand coup de poing lui briser le cerveau,

Pour lui faire savoir, roulant dans la poussière,

Qu'il n'a pu lire en "prose, hier ", ce qu'en ma colère,

J'ai brûlé comme vers depuis deux jours entiers; 
Mais réprimant soudain des transports trop altiers, Crainte de m'attirer quelque duel perfide, Dont, je le dis tout haut, je ne suis pas avide, Je change de visée, et d'un air cle bonté:

Monsieur, lui dis-je alors, je suis vraiment flatté Que vous ayez trouvé mes vers... ma prose bonne.. À ce grand mot de vers qui par mégarde sonne, Je vois pâlir le sire et froncer le sourcil, Ne me laissant plus voir que son honteux profil, Il croit que j'ai compris seulement à son dire Que d'une ouvre défunte il n'avait rien dû lire; Nous restons un instant déroutés, et des yeux Querellons tour à tour et la terre et les cieux, Quand mon haut protecteur, pour se tirer d'affaire, (Chose qu'autant que lui mon cœur brûlait de faire!) Me dit troublé, je pars, et, me pressant la main, Je vous reporterai votre pièce demain.

Ne vous dérangez pas, dis-je, j'irai la prendre; Mais non, dispensez-vous, Monsieur, de me la rendre, Procurez-moi l'honneur de vous en faire don. Puisque vous le voulez, j'accepte sans façon, Dit-il, et vous rends grâce, - Il n'en vaut pas la peine, Et de l'occasion je bénirai ma veine, Si vous daignez, Monsieur, la relire à loisir, Comment donc! c'est toujours avec nouveau plaisir, Mais je pars. Il partit, et sur ma Tragédie, C'est ainsi qu'entre nous finit la comédie; Croyez que de ceci le principal est vrai, (Noble encouragement pour mon premier essai!) ${ }^{16}$

De semblables stratégies bancales accompagnent ses prouesses ultérieures dans le métier des lettres. Comprenant à la lettre qu'il fallait introduire dans la littérature les choses et les idées du jour, Gagne cherche à faire des épopées à partir des catastrophes de l'heure (accidents de chemin de fer, incendies). Mais les faits divers ont désormais leur propre espace dans les pages des journaux et la poésie épique est d'un temps irrémédiablement révolu. Or, ces échecs répétés, Gagne va, d'une certaine manière, les transformer en formule gagnante. Que cela soit de

16 Paulin Gagne, Le Suicide, ou Cris de désespoir, de baine, de défaite, et chants d'espérance, d'amour, de triomphe, etc. Poème dramatique, Paris : Chez l'Auteur, rue Beaujolais-des-Tuileries, $\mathrm{n}^{\circ} 4$; Ledoyen, libraire, galerie d'Orléans, $\mathrm{n}^{\circ} 31$; Et chez plusieurs autres Libraires de Paris et des départemens, 1841, in-12, p. 11-15. 
86

gaieté de cour ou de parfaite conscience, il est permis d'en douter, mais le fait est là : à la différence de centaines et de centaines d'aspirants à la publication et à la reconnaissance publique, il va publier et devenir un homme connu, non seulement à titre de pitre sociopolitique du second Empire, mais aussi à titre de poète dont il est de bonne guerre de rire, car il s'y prête mieux qu'aucun autre.

De quoi et de qui rit-on quand on parle de Gagne? De sa prolixité, d'un humour qu'il aurait et qui serait ingénu, de certains de ses tics stylistiques comme sa manie d'utiliser pléthoriquement le préfixe "archi-" et le suffixe "-ide". L'éclipse fait son miel de ce menu parodique:

\title{
ARCHICARTE-PROCLAMATION
}

du citoyen Gagne, archiphilanthropophage

aux électeurs de la France et du monde

\begin{abstract}
ARCHI-MENU
Potage: Purée de diabolocrates.

Entrées: L'architête à la barigoule de Charenton. - Buisson d'orteils aux ognonides.

Rotis: L'archicarcasse aux navets. - Archicuissots à l'Abd-elKadérid.
\end{abstract}

Légumes : Monopanglottes archisautés.

Salade: Archibarbe.

Desserts: Éclairs d'archigénie.- Archifromagide.

Vins : Château-Bicêtre. - Architokai. ${ }^{17}$

Le second motif générant les assauts d'esprit des journalistes et des chroniqueurs est l'obséquiosité, le respect, le conformisme institutionnel de Gagne. Il n'est pas d'écrivains jouant plus le jeu du métier des lettres que lui. L'auteur de L'Unitéide écrit aux journaux, se présente pièce en mains aux directeurs des théâtres ${ }^{18}$, propose ici et là textes et ouvres, joue au critique, adresse des pétitions, demande des récompenses pour sa contribution au pro-

17 L'éclipse, deuxième année, $\mathrm{n}^{\circ} 68$ (9 mai 1869), p. 1. Ce texte figure sur la page de couverture de la revue, inséré dans une caricature de Job représentant Gagne s'autophilanthropophageant, nu dans une marmite qui est sur le feu.

18 La plupart des pièces de thêâtre de Gagne possède quelques centaines de rôles, quand ce n'est des légions de figurants. Mais Gagne rassure toujours les directeurs de théâtre à sa façon: il leur affirme qu'il peut lui-même jouer une kyrielle de rôles à lui tout seul. 
progrès des lettres, engage des polémiques (mais craint le duel), somme l'un ou l'autre de lui répondre, pratique une sorte de guérilla littéraire à la tête d'une bande dont il est le seul guérillero. Élise Moreau de Rus, bientôt dite madame Gagne, poétesse qui reçut quelques prix de l'Académie, subit l'influence de son époux et commit par un fâcheux mimétisme conjugal Omégar, ou le dernier homme, proso-poésie dramatique de la fin des temps en 52 cbants, précédé d'un prologue et suivi d'un épilogue par $M$. Gagne ${ }^{19}$, réponse esthétique à L'Unitéide, écrivit des romans moralisateurs pour la jeunesse et quelques ouvres de circonstance, ils organisent des conférences, tiennent un petit salon littéraire (dans un cinquième étage, près du Palais-Royal). Il serait difficile d'être plus homme et femme de lettres que cela. Mais le zèle du cancre fait toujours rire:

Le relevé des pétitions adressées à la Chambre n'est pas d'une lecture aussi aride qu'on pourrait le croire.

Les petits fascicules consacrés à ce défilé sont, au contraire, pleins de révélations inattendues.

Jugez-en par ce simple extrait:

"321. - Le sieur Gagne, avocat à Paris, demande une récompense nationale pour ses travaux littéraires."

$\mathrm{Au}$ moment où nos jeunes lauréats préparaient leurs fronts à recevoir la couronne classique de papier peint, $M$. Gagne aura senti les tempes lui démanger.

Il te faut ta couronne, ô Gagne!

Et une couronne nationale encore...

Eh bien! c'est trop juste. Le pays paye assez cher un tas de gens qui l'ennuient pour faire sans regret un petit sacrifice en faveur de quelqu'un qui l'amuse.

Une seule chose m'êtonne, c'est qu'une récompense suffise à l'auteur de la Monopanglotte.

Pour l'archi-poète, ce n'était pas trop d'une archi-récompense.

Je tourne deux pages et je retombe sur ce nouvel appel:

"341.- Le sieur Gagne, avocat à Paris, demande une récompense nationale pour ses travaux littéraires."

Avis à nos législateurs.

M. Gagne s'impatiente. ${ }^{20}$

Gagne occupe de la sorte la place de pitre littéraire par excellence. Le joyeux drille ne doit cependant pas sortir du rôle

19 Paris, Didier, et l'auteur, 1859 , in-18, xxv-405 p.

20 [Paul Parfait], "Chronique du jour ", Le charivari, 1er août 1876, p. 167. 
88

que l'institution et la doxa sont prêtes à lui accorder en échange de sa notoriété pittoresque, sinon gare. Gagne fit quelquefois l'expérience de cette limite lorsqu'il rencontra sur son chemin des plumes consacrées au sein de l'instance critique, comme celle d'Alphonse Karr, romancier de Sous les tilleuls et de Geneviève, auteur d'un Voyage autour de mon jardin et d'un Livre de bord, apprécié pour la qualité de son esprit piquant (Les guêpes) et pour sa carrière de journaliste. Sur le ring littéraire du milieu du siècle, Karr devant Gagne, c'est un combat disproportionné. Et pourtant, dans La maison close, à la fin d'un long article animé de ce persiflage narquois et de cette ironie amusée qui servirent de passe-droit stylistique à la majeure partie des littérateurs ayant pignon sur rue, Karr pourfend Gagne avec férocité sous prétexte que ce dernier aurait, au milieu de l'océan de L'Unitéide, égratigné Lamartine et Hugo:

Mais je m'arrête ici. M. Gagne m'apparaît sous un jour nouveau: je l'avais pris jusqu'à présent pour un de ces demi-fous, si communs dans le monde, qui sont heureux et qui égayent les autres, et je pensais vous réjouir et lui faire plaisir en vous parlant de lui.

Mais M. Gagne n'est pas seulement un grotesque: que $M$. Gagne flatte les puissants du jour, libre à lui; qu'il nous montre une suite de quatorze Néopolans, je ne compte pas faire sur cela la moindre observation; mais qu'il essaye d'insulter les plus grands noms dont la France s'honore, qu'il choisisse surtout ceux qui sont malheureux, qu'il ne respecte ni l'exil d'Hugo, ni la pauvreté de Lamartine! Quoique le souffle manque à ce pauvre homme, quoique, voulant insulter, il n'arrive qu'à injurier, il n'en est pas moins vrai que je ne ris plus de ses vingt-cinq mille vers; - je repousse le volume du pied et je dis: un crapaud sans venin est néanmoins un crapaud, et je détourne les yeux, et je fais essuyer la place que le poëme de M. Gagne a occupée sur ma table pendant deux jours ${ }^{21}$.

Tantôt drôles, tantôt pathétiques, les aventures du "mauvais poète prolixe." qui faisait rire de lui n'ont a priori qu'un intérêt secondaire et ne concernent que quelques érudits du troisième type ou que de pervers amateurs d'une conception "charmante" de l'histoire. Pourtant, l'examen de la carrière d'un littérateur comme Gagne conduit à poser des questions auxquelles répondre devrait permettre une meilleure connaissance des formes et 
procédures de l'illégitimation culturelle au $\mathrm{XIX}^{\mathrm{e}}$ siècle, des mécanismes de déclassement à l'œuvre dans l'Institution littéraire, ainsi que de la constitution et de l'histoire des représentations sociales reliées à l'originalité, à l'excentricité littéraire et à la folie (littéraire et autre).

Gagne en effet n'est que la pointe d'un gros iceberg. Descendant de cet abbé Le Senne si bien mis en valeur par les travaux de Robert Darnton ${ }^{22}$, petit-fils du neveu de Rameau mais sans oncle pour fixer avec flamboyance son ressentiment, l'auteur de L'Unitéide est l'un de ces innombrables plumitifs, écrivailleurs, littérateurs qui formèrent l'infanterie et le prolétariat des lettres. Estce l'aberrance esthétique de Gagne qui le rend visible? Oui, mais pas seulement. En fait, Gagne n'est sorti de l'anonymat ${ }^{23}$ où demeurèrent tant de littérateurs de son temps que parce que la rumeur peut le transformer en personnage burlesque. Son ouvre ne fait événement - il est le premier à s'en rendre compte que parce qu'il accepte à son verbe défendant de se faire anecdote, d'alimenter par ses textes, ses démarches, ses prises de parole publiques, ses journaux, ses candidatures électorales, l'infinie chronique anecdotique du $\mathrm{xIx}^{\mathrm{e}}$ siècle. Le récit d'historiettes et d'anecdotes littéraires pittoresques est en effet un véritable sport national, étroitement lié au développement de la presse boulevardière, et qui n'est pas sans effet sur l'autonomisation (très) relative de l'espace littéraire. La position de Gagne sur l'échiquier des lettres résulte autant de sa propre inaptitude à trouver les coups gagnants que des mécanismes de marginalisation à l'ouvre dans le champ littéraire. Ce poète qui fait rire est un poète dont on fait rire. Sa présence régulière dans les journaux et les revues est un feuilleton où se dit et se redit ad libitum le pathétique drôle de sa stratégie d'échec. Mais il faut voir que cette mutation du mauvais poète en figure pittoresque trouve sa raison dans le fait que la stratégie d'échec en question est éminemment acceptable et recevable, car elle dissimule sous le couvert de la folie douce ("un de ces demi-fous, si communs dans le monde, qui sont heureux et qui égayent les autres", écrit Karr) des théories d'autres trajectoires littéraires, moins drôles, misérables, bêtement ternes. Tout discours social produit par une société donnée a de la sorte

22 Cf. Robert Darnton, Gens de lettres, gens du livre, traduit de l'américain par Marie-Alyx Revellat, Paris, Editions Odile Jacob, 1992, 379 p.

23 Il dépense lui-même une énergie colossale pour se sortir de l'anonymat. 
90

besoin d'élire et de désigner des cas qui agissent comme autant de trompe-l'œil. Gagne, et ses textes, avaient tout ou finirent par avoir tout ce qu'il fallait pour entrer dans le costume du clown lettré et devenir un "homme du jour " en négatif. L'esprit dont des dizaines et des dizaines de commentateurs firent assaut à son égard est une sorte d'écran de fumée doxique: sous la verve spirituelle qui entoure le cas Gagne, c'est l'arbitraire même de la légitimité culturelle qui s'efface et se dissimule.

La catégorie qui permettra de désigner le cas, et de lui assimiler ses semblables, est celle de la "folie littéraire". Corrélée au paradigme plus large de l'originalité, elle jouxte d'autres catégories telles l'excentricité, le détraquement ou la singularité ${ }^{24}$, et s'offre en conjoncture comme une inversion de celle du génie. $\mathrm{Si}$ l'enthousiasme du génie est le comble de la valeur parce qu'il lui permet de révéler l'époque à elle-même, le "fou littéraire " du xIX siècle est quant à lui habité par un enthousiasme incommunicable, autarcique, institutionnellement autistique; il incarne la version lettrée du "monomaniaque". Cette catégorie est pourtant d'évidence une pure fiction. Il suffit de parcourir plusieurs ouvrages consacrés à la question pour apercevoir que le florilège d'originaux qu'on y rassemble est d'une hétérogénéité qui défie tout classement, en sorte qu'il vient à l'idée que la seule raison objective de l'existence de cette catégorie tient à son effet pragmatique: elle sert premièrement à désigner la frontière du bon goût, du moral, du raisonnable et à conforter l'adéquation esthétique de la vraisemblance et de la mimésis; deuxièmement à donner aux aliénistes des exemples culturels de leur définition larvaire du fou, celle de quelqu'un qui a cru trop fort à une idée, qui est victime d'une et d'une seule "manie".

Le "fou littéraire" n'a cependant que le droit et que le devoir afférents à sa fonction de bouffon de la société des gens de lettres. L'éreintage d'Alphonse Karr cité ci-dessus l'indique clairement: quand on s'appelle Gagne, on reste à sa place, on ne peut s'en prendre aux grands auteurs, on doit demeurer politiquement et littérairement inoffensif. Et cette fausse audace de Paulin Gagne:

j'ose dire, cependant, que si mon poême La guerriade était signé des noms de Lamartine, Dumas, Victor Hugo, Théophile

24 L'histoire de ces catégories et de leur émergence, ainsi que l'étude de leurs corrélations restent à faire. 
Gautier et autres poètes justement célèbres, les lecteurs et les journalistes diraient que La guerriade est un poême épique de génie $^{25}$,

pour irréaliste qu'elle soit, est au moins révélatrice, par antiphrase en quelque sorte, de sa conscience du fait que sa réputation le précède. Le personnage dépeint par la presse a désormais pris toute la place et, quand bien même La guerriade serait un grand texte, elle serait par avance lue comme le texte d'un amuseur public. Il est raisonnablement probable que Gagne disjoncta un peu plus fort que la moyenne de ses semblables, même s'il ne renvoya jamais la réplique à Shakespeare en faisant tourner des tables, mais il est plus intéressant d'observer qu'il s'est aussi conformé à cette image du délirant pittoresque. Cette dernière jouait un rôle de renforcement des hiérarchies institutionnelles, car le " fou littéraire" légitime par un effet de retour aussi bien celui qui le déclare tel que le grand auteur qui a su transformer sa "manie" en "génie". Comme tous les grands bouffons, Gagne eut droit à de justes attentions post mortem:

Encore une physionomie originale qui disparaît.

Gagne, l'avocat universel, l'archi-pontife du monde, l'auteur de l'Unitéide, vient de mourir.

Il ne manquait rien à sa gloire, car il n'est pas de journal, grand ou petit, qui n'ait inséré ses inqualifiables élucubrations. ̀̀ peine une publication nouvelle se montrait-elle à l'horizon, qu'on voyait accourir vers elle un vieillard grand et sec, aux longs cheveux, la tête ensevelie dans un énorme faux-col. C'était Gagne qui venait apporter ses souhaits de bienvenue à la feuille nouvelle. Et, tandis qu'une foule de jeunes littérateurs soupirent après les honneurs de l'impression, et voient refuser leur copie, Gagne, plus heureux, avait accès partout. Il est vrai qu'il travaillait uniquement pour la gloire et que, jamais il n'a réclamé à un journal le prix de sa collaboration toute bénévole. Comme homme politique, il ne changea jamais d'opinion; ce lui eût été, du reste, fort difficile, car cet homme étrange, ce patriarche fantaisiste, avait toutes les opinions à la fois. Ce fut lui qui, somme toute, fut le premier organisateur de la "fusion", - mais d'une fusion générale de tous les partis politiques s'embrassant fraternellement pour fonder le "quinquevir salvat".

Avant tout, cet original était patriote. On s'en souvient, ce fut lui qui, pendant le siège, proposa de tuer tous les vieillards et

25 Paulin Gagne, La guerriade..., p. 5-6. 
92

de les manger. Il s'offrait le premier en holocauste sur l'autel de la patrie.

Gagne était un monomane, mais cette douce monomanie cachait peut-être un grand bon sens. Et, d'ailleurs, qui n'a sa folie en ce monde? Il avait raison le poète qui disait

Tous les hommes sont fous, et qui n'en veut point voir

Doit rentrer dans sa chambre et casser son miroir ${ }^{26}$.

Par-devers l'institution des lettres, il avait accompli sa mission: il avait été le mauvais poète idéal, celui qui faisait rire de lui.

26 E. Villiers, “Choses du jour ", Le charivari, 29 août 1876, p. 187-188. 
Pierre Popovic
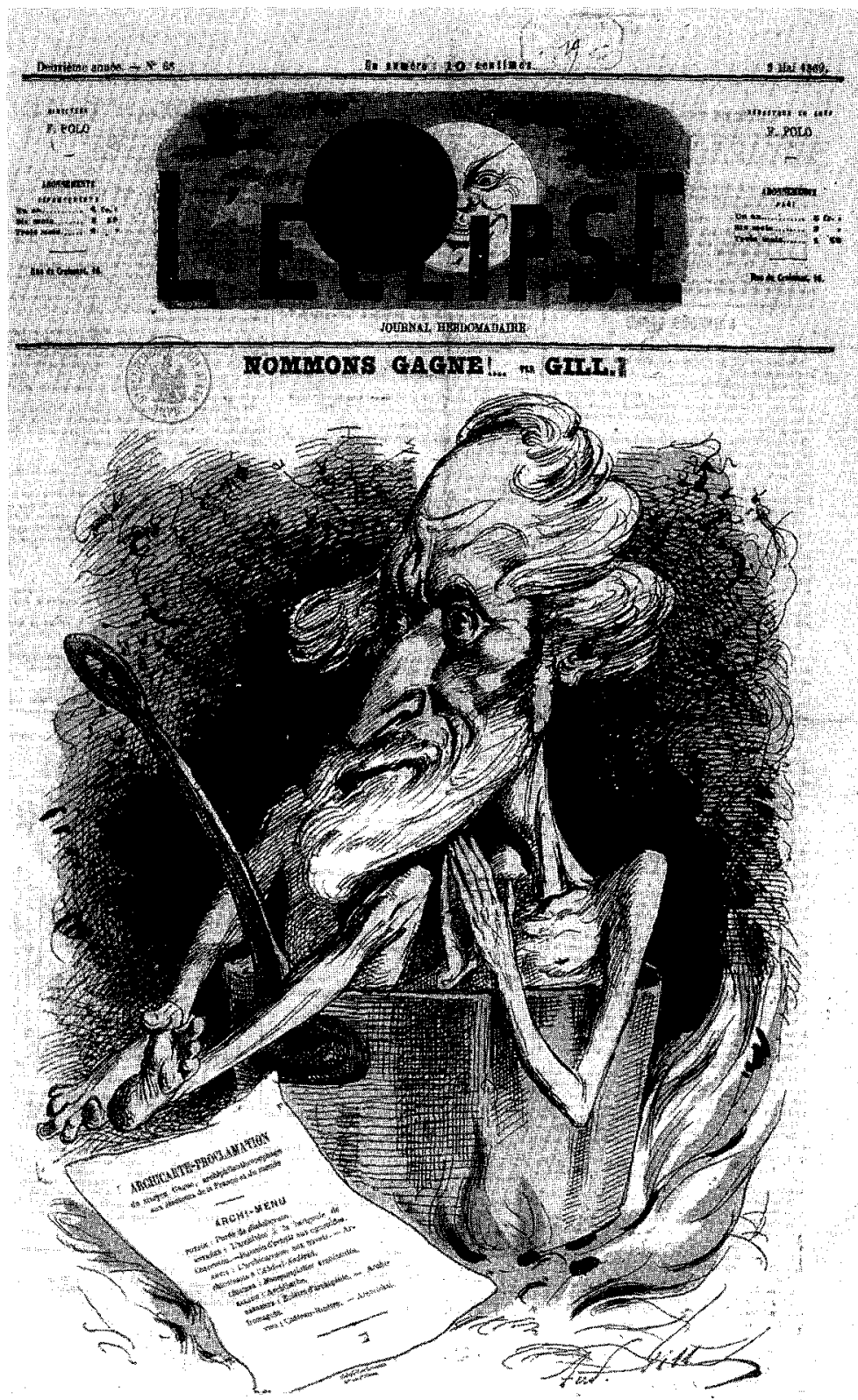
Tangence 53

94
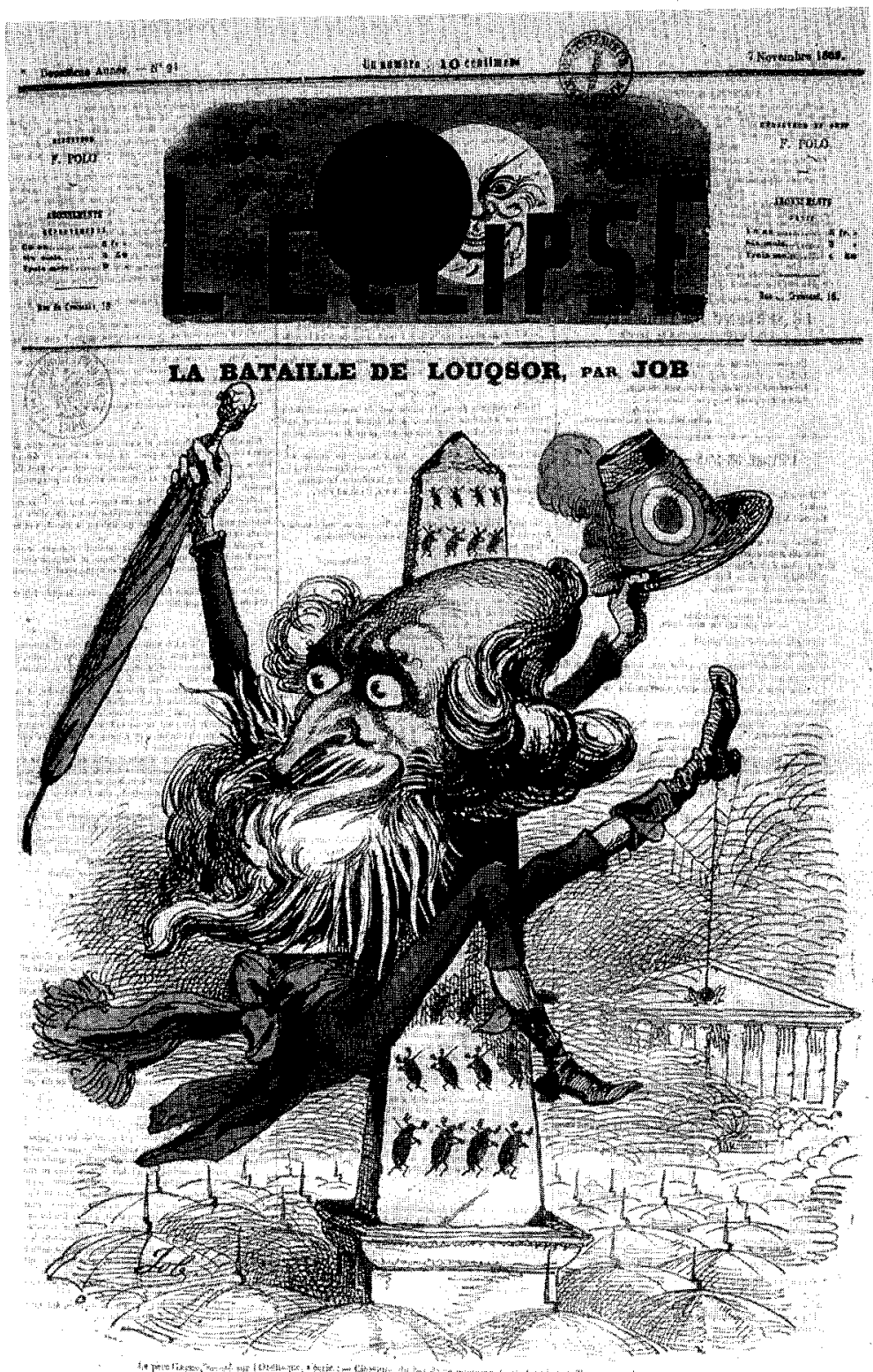


\section{ANNEXES}

\section{Octave Delepierre, sur L'Unitéide:}

C'est un poème de 724 pages, en $8^{\circ}$ (1858), composé par M. Paulin Gagne, avocat et auteur d'autres compositions poétiques.

L'Unitéide, ou la Femme-Messie, est ainsi que le dit l'auteur, un poème universel en douze chants, et en soixante actes, dont l'action se passe en l'an de grâce 2000 de l'Ère Chrétienne, et dont chaque chant forme un tout complet.

On y rencontre la plus bizarre agglomération de noms fantastiques et de vers saugrenus, que puisse inventer le cerveau humain.

La table des matières mériterait de trouver place dans un recueil de facéties.

Dans le drame on voit parler et agir tour à tour l'Âne-Archide, Fille du despotisme et de la Liberté.

Demounas, le précurseur de l'Antéchrist, la Panarchie, la Dive Infania, le Bceuf Apis, l'Archimonde et son illustre épouse La Presse, la Pataticulture, et vingt autres personnages tous plus extraordinaires les uns que les autres. Mais ces noms bizarres ne sont rien en comparaison de la bizarrerie des vers et des idées, qu'il serait difficile de faire comprendre, à qui n'a pas le livre sous les yeux. Essayons d'en donner une esquisse.

Tournant en anagramme les noms des réformateurs socialistes modernes, l'auteur les met en présence de l'Âne-Archide qui leur dit: -

Parlez donc;

Sans dormir, si je puis, j'écouterai vos rêves.

Parlez Pierre Xourel, Nodourp, Urdel-Nillor,

Louis Cnalb, Georges Nas, Narrédisnoc sans or,

Tebac, Oguh sans peur, et vous tous grands apôtres

Qui prétendez marcher sur la tête des autres.

Alors le poète expose, par leur bouche, les divers systèmes de ces Messieurs, (tels qu'il les comprend, bien entendu) dans une série de vers incroyables.

Le premier chant se termine par l'entrée de la Femme-Messie à Paris. 
96

Le second chant nous présente une partie des mêmes personnages, augmentés des ambassadeurs du Soleil et de la Lune, des habitants des astres, de l'Aurithéocratie, de la Ratiothéie, etc. Ici l'extravagance de la mise en scène dépasse encore ce qui précède. La Comète Trouble-tout a une discussion avec la Tatiothéie. L'auteur en l'introduisant, a soin de décrire son costume: "Elle est couverte d'une immense Tullillusionine (?) qui jette des éclairs, coiffée d'une chevelure de serpents rouges, et pourvue d'une queue aux feux les plus ardents. Elle chante la chanson suivante, appelée le Galop de la Comète, sur l'air: Les dëfenseurs de la Religion:-

Peuples, je viens sonner l'heure dernière

Sur les clochers de l'immense univers!

Déjà la Mort creusant la vaste bière,

Du grand convoi fait les apprêts divers;

Peuples, tremblez, vous n'avez plus de tente,

Adressez-vous le plus touchant adieu!

Peuples, tremblez devant ma queue ardente!

Peuples, roulez dans le chaos de feu!

On peut s'imaginer quelles luttes s'établissent entre les personnages, après un pareil début.

Au chant III, La Socialiforce tient un long discours à ses partisans, qu'elle termine ainsi :-

Je fonde pour toujours les âges d'or du ventre,

Dont la raison moderne élargit le doux centre;

C'est le ventre qui fait les révolutions,

Et les créations et les destructions.

Des ventres vides font toute nuit de tonnerres;

Des ventres bien garnis sortent toutes lumières;

Enfin les ventres creux ne valent jamais rien.

Donc je veux les remplir, pour qu'ils me chantent bien.

Nous allons, chers amis, sans perdre une seconde,

Préparer des festins qu'admirera le monde.

Le chant Vme, dont la scène se passe partout où l'on voudra, dit le texte, se compose d'idées si peu décentes qu'il serait ridicule d'en donner des extraits.

L'acte $38 \mathrm{me}$ du chant VIIIme, se développe dans un vaste champ de pommes de terre, et la Pataticulture ouvre la scène par un discours de 72 vers, d'autant plus singuliers que, comme nous le démontrerons tout à l'heure, ils sont écrits très sérieusement: - 
Peuples et Rois, je suis la Pataticulture, Fille de la nature et du siècle en friture;

J'ai toujours adoré ce fruit délicieux

Que, dit-on, pour extra, mangeaient jadis les Dieux.

La tirade se termine par ce vers: -

Dans le [sic] pomme de terre est le salut de tous!

On croirait qu'il est difficile d'aller plus loin dans le grotesque; mais à l'acte suivant, dont la scène, dit le texte, se passe partout, La carotticulture tient aux rois et aux peuples un discours qui l'emporte sur le précédent. On y trouve la parodie de la Marseillaise, intitulée la Carotte universelle, commençant par: -

Allons, Enfans de la Carotte,

Le jour de gloire est arrivé.

Et le chœur chante: -

Aux armes, Carottiers, formez vos bataillons,

Marchons, que la carotte inonde nos sillons.

Probablement que le lecteur croira que tout ceci n'est qu'une plaisanterie; mais non seulement $M$. Gagne est très sérieux, en expliquant son ceuvre, mais il déclare en outre, dans la préface, que le vaste sujet de ce poème bumanitaire et Cbrétien, doit former la poëtique universelle de l'bumanité, et l'école de la vérité, et il s'écrie, plein d'enthousiasme: -

Telle est, telle est la Sainte et nouvelle épopée

Que de mon pur amour l'âme a développée!

Ensuite $M^{\text {me }}$ Élise Gagne, sa femme, ajoute un épilogue, où elle proclame qu'après les réformes indiquées dans le poème:-

L'abondance parvint à chasser la misère,

Et le bonheur des cieux habita sur la terre.

L'ensemble prouve, en un mot, que M. Gagne a employé toutes les ressources de son intelligence pour écrire ce chefd'œuvre, et si le lecteur est tenté de rire, c'est qu'il ne comprend pas l'extrême profondeur de la pensée qui enfanta ce poème.

N'est-ce pas bien le cas de dire, avec le sieur de Longval "Lorsque cette Méduse (la manie) s'est une fois glissée dans le cerveau, elle sait si bien offusquer l'imagination, pervertir les pensées, transporter l'esprit, et corrompre la raison, que par son 
98

moyen les actions et les paroles des hommes se tournent en extravagances. " 27

\section{Bibliographie des écrits de Paulin Gagne}

En plus de L'Unitéide, l'œuvre de Paulin Gagne comprend les titres suivants: Le Suicide, ou Cris de désespoir, de baine, de défaite, et chants d'espérance, d'amour, de triomphe, etc. Poème dramatique, Paris: Chez l'Auteur, rue Beaujolais-des-Tuileries, $\mathrm{n}^{\circ} 4$; Ledoyen, libraire, galerie d'Orléans, $\mathrm{n}^{\circ} 31$; Et chez plusieurs autres Libraires de Paris et des départemens, 1841, in-12, 148 p.; À la famille royale, à la France. Le martyre des rois, ou La vie et la mort de Mgr le duc d'Orléans,... ses funérailles à Paris et à Dreux, son apotbéose, la séance royale, le discours du Roi, poëme, odeélégie... suivi de nombreux articles en prose, [Paris], I. Rousset, 1842, in-18, 85 p.; Catastophe du chemin de fer. Au profit des victimes. Le délire, monologue en vers, Paris, Ledoyen, 12 mai 1842, in-8, 16 p.; La Gagne-monopanglotte, ou Langue unique et universelle, formée de la réunion radicale et substantielle de toutes les langues mères... et l'Empire universel, poème en 10 chants et dont il est seulement donné ici quelques fragments, Paris, I. Rousset, 1843 , in-8, 16 p. (À tous les souverains, aux savants, aux philosophes, aux législateurs, aux poètes et à tous les peuples du monde); L'Océan des catastrophes, poème sur les désastres du 8 février... avec des notices en prose sur le tremblement de terre d'Antigoa et de Liverpool, suivi de l'incendie de Hambourg, poème, et sur la nouvelle comète de 1843, Paris, I. Rousset, 1843, in-16, 48 p.; À l'union de la France et de l'Espagne. Le triomphe de l'amour royal ou le mariage de S.A.R. le duc de Montpensier et de S.M. la reine d'Espagne, poème cantate lyrique, Paris, l'auteur, 1846, in-16, 16 p.; Voyage de S.A.I. Napoléon, ses discours à Lyon et à Bordeaux, itinéraire et entrée à Paris. Vive l'Empire et vive l'Empereur! cbant lyrique et final; et l'Unitéide des peuples, ou la Femme messie et unitrice sauvant le monde, qui prend son nom, par l'Unité napoléonienne. Recueils de poèmes et chants populaires, Paris, Ledoyen, [1852], in-18, 40 p.; Voyage de S.A.I. Napoléon, ses discours à Lyon, à Bordeaux, et à Paris. Vive l'Empire et vive l'Empereur! chant lyrique et final; et l'Unitéide des

27 Octave Delepierre, Histoire littêraire des fous, London, Trübner \& Co., 60 , Paternoster Row., 1860, 181 p., p. 61-65. 
peuples, recueils de poèmes et chants populaires, Paris, Ledoyen, [1852], in-18, 36 p.; Le théâtre du monde, joumal et cours littéraires et artistiques (avec la collaboration de Mme Élise GagneMoreau de Rus), Paris, place de Laborde, janvier 1854[- décembre 1857], 4 vol., in-8; Le Journalophage, ou le Mangeur de journaux, satire anti-journal... paraissant quand il peut, Paris, l'auteur, [1858], in-8, nos 1-7.[Les numéros $2-7$ portent en outre: $5^{\mathrm{e}}$ année comme successeur du "Théâtre du monde" - les numéros 6-7 ont pour titre: Le Journalophage et l'Uniteur, journal des journaux, réunis par $M$. Gagne] - 1. Le Journal-antéchrist; 2 . Le Journal chronicritique annonce; 3. L'Amour de l'immortalité; 4. La Revue de l'unité universelle; 5 . Les Poètes et les journaux; 6. La Fête du journalophage; 7. L'Art de dompter les journaux, les journalistes et les chevaux; Les vendeurs du temple et la voix du salut, Paris, l'auteur, 1859 , in- $8^{\circ}, 16$ p. [Rp.3466 (À tous les peuples du monde - Affaire du jeune Mortara); L'bistoire des miracles renfermant une dédicace à Mme Gagne, un préambule bistorique, l'bistoire de ma mort, les mémoires de ma vie miraculeuse et le bonbeur du crucifiement, Paris, l'auteur, 1860, in-18, 72 p. ; L'Uniteur du monde visible et invisible, journal universel de journaux et de l'unité... Miracles et révélations opérés et faits en la personne de $M$. Gagne par l'Esprit divin, Paris, impr. de Dubuisson, $\mathrm{n}^{\circ} 1[-4]$, janvier[-avril] 1860, in-fol., 16 p.; Le Tribut d'amour à M. Desgenettes, curé de Notre-Dame des Victoires, Paris, M. Moreau, [1860], in-18, 17 p.; La Comète de l'Antéchrist. présentée à l'Académie des sciences le 8 juillet 1861, Paris, impr. de Dubuisson, [1861], in-8, 4 p.; Le Calvaire des rois: Louis XVI, Marie-Antoinette, Elisabeth et Louis XVIII, martyrs et christs de la royauté, régo-tragédie épique, bistorique et nationale en 5 actes et en vers, Paris, F. Henry et l'auteur, 1863, in-8, iv-300 p.; Le Congrès sauveur des peuples et des rois, salutéide ou poème-opéra de salut à l'avenir, bistorique, national et universel, en 24 chants-actes dialogués destinés au théâtre et en vers, Paris, F. Henry et l'auteur, 1864, in-8, 184 p.; L'Anarchiade de la décentralisation, archi-drame flagellateur en 5 éclats, joué sur tous les théâtres du monde, Paris, l'auteur, [1865], in-8, 4 p.; Les deux luxes des hommes et des femmes, luxéide, drame prostitutionnicide et luxicide en 3 éclats, joué sur tous les théâtres du monde, précédé d'une préface et suivi d'un épilogue, Paris, impr. de Jouaust, [1865], in-8, 16 p. ; Les deux sueurs froides et chaudes, produites par "Les deux sceurs " de M. Émile de Girardin, archidrame vengeur, Paris, l'auteur, [1865], in-8, 4 p.; L'Archi-soleil, grand archi-journal des éclats universels et uniteur de 
100

l'avenir (Rédacteur en chef: M. Gagne), $\mathrm{n}^{\text {os }} 1-12,8$ octobrel-24 décembre] 1865, Paris, impr. de Jouaust, in-4, 48 p.; La grévéide, drame grévicide universel des grèves, en 5 éclats, précédé d'une prêface de salut et suivi d'un épilogue d'amnistie, Paris, Impr. de Jouaust, [1865], in-8, 16 p. ; La Sataniade du spiri-satanisme, archi-drame spiriticide en 5 éclats infernaux, joué sur tous les théâtres du monde, précédé d'une préface miraculeuse, Paris, tous les libraires, [1865], in- $8^{\circ}, 4$ p.; Le supplice d'un mari, drame réél et universel en 5 tableaux-scènes, joué sur tous les thêâtres du monde, précédé d'un prologue-préface, [Paris], tous les libraires, [1865], in-8, 16 p.; L'Oracle, panglotte universel de toutes les langues dont il donne la clef... suivi de 20 oraisons dominicales, de mots en 44 langues, de poésie au Palais Soleil de l'Exposition et d'un Pater en langue universelle, Paris, tous les libraires, [1866], in-4, 8 p.; L'Expositionnide, déesse des expositions, logoside-poème dramatique de l'Exposition universelle de 1867 en 5 actes et en vers, [Paris], tous les libraires, [1866], in-8, 16 p.; L'Unité, journal universel et pantoglotte de l'Avenir, Paris, impr. de Jouaust, $\mathrm{n}^{\circ} 1$ 13], octobre 1867[-octobre 1868], in-4, 52 p.; L'Archi-monarque sauveur de l'Italie et du monde entier, suivi du Chant de l'archimonarque, Paris, l'auteur, 1868, in-4, 4 p.; Le vélocitêteGagne...La Vélocitéide... Le chant de triomphe du vélocitête, Paris, impr. de Jouaust, [1870], in-fol.; La Républiquéide, empire, royauté, seul gouvernement définitif de salut... dirigé par le Trium-vir-salvat de Thiers, ou de Hugo, ou du duc d'Aumale, ou de Gambetta, et de Napoléon III et de Henri V, vélocitête-poèmeopéra dramatique en 5 gestes à réveils, Paris, l'auteur, 1872, in-8, 16 p.; La Guerriade, déesse de la guerre, poème épique de la guerre étrangère, civile, politique et morale en 12 chants, Paris, tous les libraires, 1873, in-18, 108 p.; Les cris de l'âme de Napoléon III (discours extrait de "La Guerriade", poème en 12 chants), Paris, l'auteur, 1873, in-8, 4 p.; L'beure de Dieu, étrennes de la prorogation, poésie de l'avenir républicaine-impériale-royale à réveils ou répétitions de vers, Paris, l'auteur, 1874, in-8, 4 p.; L'Archi-monarquéide, ou Gagne premier, archi-monarque de la France et du monde, par la grâce de Dieu et de la volonté nationale, poème-tragédie-comédie-drame-opéra épique en 5 actes et 12 chants, avec chceurs, joué sur tous les thêâtres du monde, précédé d'une préface et d'un prologue, et suivi d'un épilogue, Paris, tous les libraires; et chez l'auteur, 1876, in-18, 108 p. Dans la confrérie des "fous littéraires", Gagne se distingue par l'insigne honneur d'avoir eu certains de ses textes réédités au vingtième 
siècle. outre "L'Oraison dominicale en langue universelle [...] somptueusement imprimée à 32 exemplaire par Alain Guinhut pour G[ustave]-A[rthur] Dassonville" (Alain Blavier, Les fous littéraires, p. 59, n. 81), il faut citer: Neuf lettres, précédées d'une profession de foi, d'un quatrain sur le salut de l'Italie, et suivies de la Constitution philantbropophagique, telle qu'elle a paru dans " L'Unité "d'auril 1868, par M. Gagne, avocat, ancien bâtonnier du barreau de Montélimar, rédacteur en chef du journal L'Unité, auteur de L'Archimonarquéide ou Gagne 1er, archimonarque de la France et du monde, ouvrage illustré d'un portrait de M. Gagne d'après la pboto de $M$. Laurent, de la reproduction d'une caricature en couleurs et de trois fac-simile. Préface de Gustave Artbur Dassonville. Postface d'André Blavier, À Verviers, au bureau de la revue Temps mêlés, 1960, 29 p. 